\title{
Probing top charged-Higgs production using top polarization at the Large Hadron Collider
}

\author{
Katri Huitu, ${ }^{a}$ Santosh Kumar Rai, ${ }^{b}$ Kumar Rao, ${ }^{a}$ Saurabh D. Rindani ${ }^{c}$ and \\ Pankaj Sharma ${ }^{c}$ \\ ${ }^{a}$ Department of Physics, University of Helsinki, and Helsinki Institute of Physics, \\ P.O. Box 64, FIN-00014 Helsinki, Finland \\ ${ }^{b}$ Department of Physics, and Oklahoma Center for High Energy Physics, \\ Oklahoma State University, \\ Stillwater, OK 74078, U.S.A. \\ ${ }^{c}$ Theoretical Physics Division, Physical Research Laboratory, \\ Navrangpura, Ahmedabad 380 009, India \\ E-mail: katri.huitu@helsinki.fi, skrai22@gmail.com, \\ kumar.rao@helsinki.fi, aurabh@prl.res.in, pankajs@prl.res.in
}

ABSTRACT: We study single top production in association with a charged Higgs in the type II two Higgs doublet model at the Large Hadron Collider. The polarization of the top, reflected in the angular distributions of its decay products, can be a sensitive probe of new physics in its production. We present theoretically expected polarizations of the top for top charged-Higgs production, which is significantly different from that in the closely related process of $t W$ production in the Standard Model. We then show that an azimuthal asymmetry, constructed from the decay lepton angular distribution in the laboratory frame, is a sensitive probe of top polarization and can be used to constrain parameters involved in top charged-Higgs production.

Keywords: Higgs Physics, Beyond Standard Model, Supersymmetric Standard Model

ARXIV EPRINT: 1012.0527 


\section{Contents}

1 Introduction $\quad 1$

2 Top polarization and the spin density matrix 3

3 Top polarization in the two Higgs doublet model 5

4 Azimuthal distributions of decay leptons $\quad 9$

$\begin{array}{lll}5 & \text { Summary } & 15\end{array}$

\section{Introduction}

The properties and interactions of the top quark, except for its mass, are not yet known in detail. With a mass close to the electroweak (EW) symmetry breaking scale and thus a large Yukawa coupling, the top quark is an excellent probe of whatever mechanism is responsible for EW symmetry breaking. In the Standard Model (SM), EW symmetry is broken through a single $\mathrm{SU}(2)$ scalar doublet, i.e, through the Higgs mechanism. However, while the SM Higgs mechanism is the simplest way to break EW symmetry, there are reasons to consider an enlarged Higgs sector [1]. Models with two Higgs doublets can generate spontaneous $C P$ violation, address the strong $C P$ problem and generate additional sources of $C P$ violation needed for baryogenesis [2]. Moreover, the most popular paradigm for addressing the gauge hierarchy problem, supersymmetry (SUSY) contains two Higgs doublets in its simplest formulation $[1,3,4]$. The spectrum of two Higgs doublet models (THDM) involves three neutral and two charged Higgs bosons. Different versions of the THDM also have different couplings of the scalars to fermions. Thus, even if scalar particles were to be discovered at the Large Hadron Collider (LHC), it is necessary to probe in detail the precise couplings to these particles to establish the underlying model and pinpoint the exact mechanism of EW symmetry breaking. Charged Higgs particles exist even in extensions of the SM which involve the introduction of a $\mathrm{SU}(2)$ triplet of scalars, which are also interesting from the point of view of obtaining a small Majorana mass for neutrinos in the type-II see-saw mechanism [5-9]. It is possible to produce a single top quark in association with a charged Higgs in such models. We study, in this work, such a process in the context of a type II THDM or SUSY models, where the up type quarks couple to one of the Higgs doublets and down type quarks couple to the other Higgs doublet [1].

The study of the top quark at the Tevatron has made use of the sample of top-antitop pairs produced in large numbers. At the LHC, there would be copious production of $t \bar{t}$ pairs, and one can think of the LHC as a top factory. While pair production would be most useful for studying many properties of the top quark, single-top production, which 
proceeds via the weak interaction, would be more suitable to study the weak sector. In particular, measurement of the CKM matrix element $V_{t b}$ can be made using single-top events. While a few single-top events have been seen at the Tevatron, at the LHC a much larger rate will be seen, and the single-top channel will be useful for a confirmation of the SM couplings for the top, and a precise measurement of $V_{t b}$.

With a large mass of $\sim 172 \mathrm{GeV}$, the top quark has an extremely short lifetime, calculated in the SM to be $\tau_{t}=1 / \Gamma_{t} \sim 5 \times 10^{-25} \mathrm{~s}$. This is an order of magnitude smaller than the hadronization time scale, which is roughly $1 / \Lambda_{\mathrm{QCD}} \sim 3 \times 10^{-24} \mathrm{~s}$. Thus, in contrast to lighter quarks, the top decays before it can form bound states with lighter quarks [10]. As a result, the spin information of the bare top, which depends solely on its production process, is reflected in characteristic angular distributions of its decay products. Thus, the degree of polarization of an ensemble of top quarks can provide important information about the underlying physics in its production, apart from usual variables like cross sections, since any couplings of the top to new particles can alter its degree of polarization and the angular distributions of its decay products. ${ }^{1}$ In this paper, we investigate the effects on top polarization in the single production of the top in association with a charged Higgs of the type II THDM or the minimal supersymmetric standard model (MSSM).

Single-top production in association with a charged Higgs can be used to probe the size and nature of the $t b H$ coupling. Apart from the cross section, the angular distribution of the top, and even the polarization of the top would give additional information enabling the determination of the $t b H$ coupling. Here we concentrate on the polarization of the top in the process, which would be a measure of the extent of parity violation in the couplings. It will be seen that polarization gives a handle on the combination $A_{L}^{2}-A_{R}^{2}$ of the lefthanded and right-handed couplings, $A_{L} \equiv m_{t} \cot \beta$ and $A_{R} \equiv m_{b} \tan \beta$ of the charged Higgs to the top where $\tan \beta$ is the ratio of the vacuum expectation values (vevs) of the Higgs doublets, in contrast to the combination $A_{L}^{2}+A_{R}^{2}$ measured by the cross section or angular distribution.

The most direct way to determine top polarization is by measuring the angular distribution of its decay products in its rest frame. However, at the LHC reconstructing the top rest frame will be difficult. In this paper, we show how the decay lepton angular distributions in the laboratory frame can be a useful probe of top polarization and the $t b H^{-}$ coupling. As will be explained in section 2, the angular distribution of the charged lepton has a special property-it is independent of new physics in the $t b W$ decay vertex, to linear order in the anomalous couplings, and is thus a pure probe of new physics in top production alone. We show that the azimuthal distribution of the lepton is sensitive to top polarization and can be used to probe the coupling parameter $\tan \beta$ in the type II THDM. This approach has been recently used to probe new physics in the case of top pair production in a model with an extra heavy vector resonance $\left(Z^{\prime}\right)$ with chiral couplings [14]. The effects of top polarization in $t W$ and $t H^{-}$production have been studied previously in [15], where the effects of 1-loop electroweak SUSY corrections have been considered; however, they do not consider top decay. Top polarization in different modes of single top production has

\footnotetext{
${ }^{1}$ For reviews on top quark physics and polarization see [11-13].
} 
also been studied in [16-18], where spin sensitive variables are used to analyze effective left and right handed couplings of the top coming from BSM physics.

This paper is organized as follows. In section 2 we discuss top polarization and outline the spin density matrix formalism, needed to preserve spin coherence between top production and decay. In section 3, we derive expressions for polarized cross sections for $t H^{-}$ production and present results for the expected top polarization in this case. In section 4 , we construct an azimuthal asymmetry involving the charged lepton from top decay which is a probe of top polarization and a sensitive measure of $\tan \beta$. Section 5 contains a summary.

\section{Top polarization and the spin density matrix}

Top spin can be determined by the angular distribution of its decay products. In the SM, the dominant decay mode is $t \rightarrow b W^{+}$, with a branching ratio (BR) of 0.998 , with the $W^{+}$subsequently decaying to $\ell^{+} \nu_{\ell}$ (semileptonic decay, BR 1/9 for each lepton) or $u \bar{d}, c \bar{s}$ (hadronic decay, BR 2/3). The angular distribution of a decay product $f$ for a top quark ensemble has the form ( see for example [11]),

$$
\frac{1}{\Gamma_{f}} \frac{\mathrm{d} \Gamma_{f}}{\mathrm{~d} \cos \theta_{f}}=\frac{1}{2}\left(1+\kappa_{f} P_{t} \cos \theta_{f}\right)
$$

Here $\theta_{f}$ is the angle between $f$ and the top spin vector in the top rest frame and

$$
P_{t}=\frac{N_{\uparrow}-N_{\downarrow}}{N_{\uparrow}+N_{\downarrow}}
$$

is the degree of polarization of the top quark ensemble where $N_{\uparrow}$ and $N_{\downarrow}$ refer to the number of positive and negative helicity tops respectively. $\Gamma_{f}$ is the partial decay width and $\kappa_{f}$ is the spin analyzing power of $f$. Obviously, a larger $\kappa_{f}$ makes $f$ a more sensitive probe of the top spin. The charged lepton and $d$ quark are the best spin analyzers with $\kappa_{\ell^{+}}=\kappa_{\bar{d}}=1$, while $\kappa_{\nu_{\ell}}=\kappa_{u}=-0.30$ and $\kappa_{b}=-\kappa_{W^{+}}=-0.39$, at tree level [11]. Thus the $\ell^{+}$or $d$ have the largest probability of being emitted in the direction of the top spin and the least probability in the direction opposite to the spin. Since at the LHC, leptons can be measured with high precision, we focus on leptonic decays of the top.

For hadronic $t \bar{t}$ production, spin correlations between the decay leptons from the $t$ and $\bar{t}$ have been extensively studied in the SM and for BSM scenarios [11, 12, 19-24]. These spin correlations measure the asymmetry between the production of like and unlike helicity pairs of $t \bar{t}$ which can probe new physics in top pair production. However, this requires the reconstruction of the $t$ and $\bar{t}$ rest frames, which is difficult at the LHC. Here we investigate top polarization in the lab. frame, which would be more directly and easily measurable without having to construct the top rest frame.

Let us consider a generic process of top charged-Higgs production and subsequent semileptonic decay of $t$ and inclusive decay of $H^{-}, A B \rightarrow t H^{-} \rightarrow b \ell^{+} \nu_{\ell} X$. Since $\Gamma_{t} / m_{t} \sim$ 0.008 , we can use the narrow width approximation (NWA) to write the cross section as a product of the $2 \rightarrow 2$ production cross section times the decay width of the top. However, in probing top polarization using angular distributions of the decay lepton, it is necessary 
to keep the top spin information in its decay arising from its production, thus requiring the spin density matrix formalism. As in [25], the amplitude squared can be factored into production and decay parts using the NWA as

$$
\overline{|\mathcal{M}|^{2}}=\frac{\pi \delta\left(p_{t}^{2}-m_{t}^{2}\right)}{\Gamma_{t} m_{t}} \sum_{\lambda, \lambda^{\prime}} \rho\left(\lambda, \lambda^{\prime}\right) \Gamma\left(\lambda, \lambda^{\prime}\right),
$$

where $\rho\left(\lambda, \lambda^{\prime}\right)$ and $\Gamma\left(\lambda, \lambda^{\prime}\right)$ are the $2 \times 2$ top production and decay spin density matrices and $\lambda, \lambda^{\prime}= \pm 1$ denote the sign of the top helicity. After phase space integration of $\rho\left(\lambda, \lambda^{\prime}\right)$ we get the resulting polarization density matrix $\sigma\left(\lambda, \lambda^{\prime}\right)$. The $(1,1)$ and $(2,2)$ diagonal elements of $\sigma\left(\lambda, \lambda^{\prime}\right)$ are the cross sections for the production of positive and negative helicity tops and $\sigma_{\mathrm{tot}}=\sigma(+,+)+\sigma(-,-)$ is the total cross section. We define the degree of longitudinal polarization $P_{t}$ as

$$
P_{t}=\frac{\sigma(+,+)-\sigma(-,-)}{\sigma(+,+)+\sigma(-,-)} .
$$

The off-diagonal elements of $\sigma\left(\lambda, \lambda^{\prime}\right)$ are the production rates of the top with transverse polarization. The top decay density matrix $\Gamma\left(\lambda, \lambda^{\prime}\right)$ for the process $t \rightarrow b W^{+} \rightarrow b \ell^{+} \nu_{\ell}$ can be written in a Lorentz invariant form as

$$
\Gamma( \pm, \pm)=2 g^{4}\left|\Delta\left(p_{W}^{2}\right)\right|^{2}\left(p_{b} \cdot p_{\nu}\right)\left[\left(p_{\ell} \cdot p_{t}\right) \mp m_{t}\left(p_{\ell} \cdot n_{3}\right)\right],
$$

for the diagonal elements and

$$
\Gamma(\mp, \pm)=-2 g^{4}\left|\Delta\left(p_{W}^{2}\right)\right|^{2} m_{t}\left(p_{b} \cdot p_{\nu}\right) p_{\ell} \cdot\left(n_{1} \mp i n_{2}\right),
$$

for the off-diagonal ones. Here $\Delta\left(p_{W}^{2}\right)$ is the $W$ boson propagator and $n_{i}^{\mu}$, s $(i=1,2,3)$ are the spin 4 -vectors for the top with 4 -momentum $p_{t}$, with the properties $n_{i} \cdot n_{j}=-\delta_{i j}$ and $n_{i} \cdot p_{t}=0$. For decay in the rest frame they take the standard form $n_{i}^{\mu}=\left(0, \delta_{i}^{k}\right)$.

Using the NWA the differential cross section for top production and decay, with inclusive decay of $H^{-}$can be written as

$$
\begin{aligned}
d \sigma= & \frac{1}{32 \Gamma_{t} m_{t}} \frac{1}{(2 \pi)^{4}}\left[\sum_{\lambda, \lambda^{\prime}} d \sigma\left(\lambda, \lambda^{\prime}\right) \times\left(\frac{\Gamma\left(\lambda, \lambda^{\prime}\right)}{p_{t} \cdot p_{\ell}}\right)\right] E_{\ell}\left|\Delta\left(p_{W}^{2}\right)\right|^{2} d \cos \theta_{t} d \cos \theta_{\ell} d \phi_{\ell} \\
& \times d E_{\ell} d p_{W}^{2}
\end{aligned}
$$

where the lepton integration variables are in the lab frame and $b$ quark energy integral is replaced by an integral over the invariant mass $p_{W}^{2}$ of the $W$ boson. $d \sigma\left(\lambda, \lambda^{\prime}\right)$ is the differential cross section for the $2 \rightarrow 2$ process of top charged Higgs production with indicated spin indices of the top. As shown in [25], by measuring the angular distributions of the decay lepton in the top rest frame (which requires reconstructing the top rest frame) analytic expressions for the longitudinal and transverse components of the top polarization can be obtained by a suitable combination of lepton polar and azimuthal asymmetries. However, as pointed out in the introduction, it would be useful and interesting to devise variables for the lepton in the laboratory frame, which are easily measured and are sensitive to top polarization. 
An important point is the possible appearance of new physics in the $t b W$ decay vertex, apart from that in top production, leading to changed decay width and distributions for the $W^{+}$and $l^{+}$. The $t b W$ vertex can be written in model-independent form as

$$
\Gamma^{\mu}=\frac{-i g}{\sqrt{2}}\left[\gamma^{\mu}\left(f_{1 L} P_{L}+f_{1 R} P_{R}\right)-\frac{i \sigma^{\mu \nu}}{m_{W}}\left(p_{t}-p_{b}\right)_{\nu}\left(f_{2 L} P_{L}+f_{2 R} P_{R}\right)\right],
$$

where for the SM $f_{1 L}=1$ and the anomalous couplings $f_{1 R}=f_{2 L}=f_{2 R}=0$. The simultaneous presence of new physics in top production and decay can complicate the analysis making it difficult to isolate new couplings of the top. However, it has been proven that the energy averaged angular distributions of charged leptons or $d$ quarks from top decay are not affected by the anomalous $t b W$ vertex. This has been shown very generally for a $2 \rightarrow n$ process and assumes the narrow width approximation (NWA) for the top and neglects terms quadratic in the anomalous couplings in (2.8) assuming new physics couplings to be small (for details see [25] and references therein). This implies that charged lepton angular distributions in the lab frame are more accurate probes of top polarization, and thus to new physics in top production alone. In contrast, the energy distributions of the $l^{+}$or the angular distributions of the $b$ and $W$ are "contaminated" by the anomalous $t b W$ vertex. In section 4 we will construct an observable using the azimuthal distribution of the charged lepton which is sensitive to the top polarization and can be measured with a large significance at the LHC.

\section{Top polarization in the two Higgs doublet model}

We consider the process of single top production in association with a charged Higgs in the type II THDM or the Minimal Supersymmetric Standard Model (MSSM). For our purposes, the model is completely characterized by two parameters, the mass of the charged Higgs $M_{H^{-}}$and the ratio of the vacuum expectation values (vevs) of the Higgs doublets $\tan \beta$. At the parton level, single top production proceeds via

$$
g\left(p_{1}\right) b\left(p_{2}\right) \rightarrow t\left(p_{3}, \lambda_{t}\right) H^{-}\left(p_{4}\right),
$$

where $\lambda_{t}= \pm 1$ is the sign of the helicity of the top. The tree level $s$ and $t$ channel diagrams contributing to the above process are shown in figure 1. As mentioned in the previous section, a study of top polarization using angular distributions of the top decay products requires computing the spin density matrix for top production and decay. We have obtained simple analytic expressions for the top production density matrix. In the type II THDM the $t b H^{-}$coupling is

$$
g_{t b H^{-}}=\frac{g}{\sqrt{2} m_{W}}\left(m_{t} \cot \beta P_{L}+m_{b} \tan \beta P_{R}\right),
$$

where $g$ is the $\mathrm{SU}(2)$ gauge coupling and $P_{L}$ and $P_{R}$ are the left and right handed projection operators respectively, $P_{L, R}=\left(1 \mp \gamma^{5}\right) / 2$. One can immediately see that at $\tan \beta=$ $\sqrt{m_{t} / m_{b}}$, the pseudoscalar part of the coupling, which is proportional to $\gamma^{5}$, vanishes and the coupling (3.2) is purely scalar. Since polarization is parity violating we expect that the 


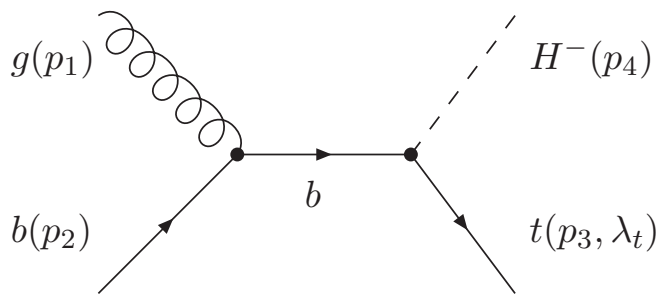

$(a)$

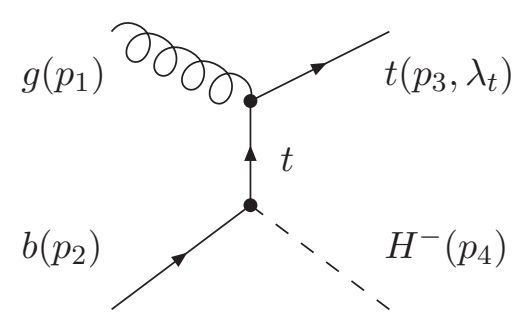

(b)

Figure 1. Feynman diagrams contributing to the top charged-Higgs production at the LHC.

polarized cross section (2.4) should vanish for this value of $\tan \beta$ and we indeed find this to be the case, as will be shown later in figure 3 .

Denoting the energy, momentum and scattering angle of the top in the parton centerof-mass $(\mathrm{cm})$ frame by $E_{t}, p_{t}$ and $\theta_{t}$ respectively and the parton level Mandelstam variable by $\hat{s}$, the diagonal elements are given by

$$
\begin{aligned}
& \rho(+,+)=F_{1} m_{t}^{2} \cot ^{2} \beta+F_{2} m_{b}^{2} \tan ^{2} \beta \\
& \rho(-,-)=F_{2} m_{t}^{2} \cot ^{2} \beta+F_{1} m_{b}^{2} \tan ^{2} \beta,
\end{aligned}
$$

where $F_{1}$ and $F_{2}$ are defined by

$$
\begin{aligned}
F_{1}= & \left(\frac{g g_{s}}{2 m_{W}}\right)^{2} \frac{1}{6 \sqrt{\hat{s}}\left(E_{t}-p_{t} \cos \theta_{t}\right)^{2}}\left\{p_{t}^{2}\left(E_{t}+p_{t}\right) \sin ^{2} \theta_{t} \cos ^{2} \frac{\theta_{t}}{2}+\left[4 E_{t}\left(E_{t}+p_{t}\right)\left(E_{t}-\sqrt{\hat{s}}\right)\right.\right. \\
& \left.\left.+2 m_{t}^{2} \sqrt{\hat{s}}+\left(\hat{s}\left(E_{t}+p_{t}\right)+m_{t}^{2}\left(E_{t}-p_{t}\right)-4 m_{t}^{2} E_{t}\right)\right] \sin ^{2} \frac{\theta_{t}}{2}\right\} \\
F_{2}= & \left(\frac{g g_{s}}{2 m_{W}}\right)^{2} \frac{1}{6 \sqrt{\hat{s}}\left(E_{t}-p_{t} \cos \theta_{t}\right)^{2}}\left\{p_{t}^{2}\left(E_{t}-p_{t}\right) \sin ^{2} \theta_{t} \sin ^{2} \frac{\theta_{t}}{2}+\left[4 E_{t}\left(E_{t}-p_{t}\right)\left(E_{t}-\sqrt{\hat{s}}\right)\right.\right. \\
& \left.\left.+2 m_{t}^{2} \sqrt{\hat{s}}+\left(\hat{s}\left(E_{t}-p_{t}\right)+m_{t}^{2}\left(E_{t}+p_{t}\right)-4 m_{t}^{2} E_{t}\right)\right] \cos ^{2} \frac{\theta_{t}}{2}\right\} .
\end{aligned}
$$

The off-diagonal elements are

$$
\begin{aligned}
\rho(+,-)=\rho(-,+)= & -\left(\frac{g g_{s}}{2 m_{W}}\right)^{2} \frac{1}{6 \sqrt{\hat{s}}\left(E_{t}-p_{t} \cos \theta_{t}\right)^{2}}\left(m_{t}^{2} \cot ^{2} \beta-m_{b}^{2} \tan ^{2} \beta\right) \\
& \times m_{t} \sin \theta_{t}\left(2 E_{t} \sqrt{\hat{s}}-m_{t}^{2}-\hat{s}+p_{t}^{2} \sin ^{2} \theta_{t}\right) .
\end{aligned}
$$

In deriving the above expressions we have neglected the kinematic effects of the $b$ quark mass but kept factors of $m_{b}$ occurring in the $t b H^{-}$coupling (3.2). Analytic expressions for the helicity amplitudes for associated $t H^{-}$production can be found in [15], where a similar convention for retaining factors of $m_{b}$ is used; our density matrix elements (3.4) and (3.7), obtained by an independent method, agree with those obtained using the helicity amplitudes of [15]. A plot of the cross section as a function of the coupling $\tan \beta$ is shown in 

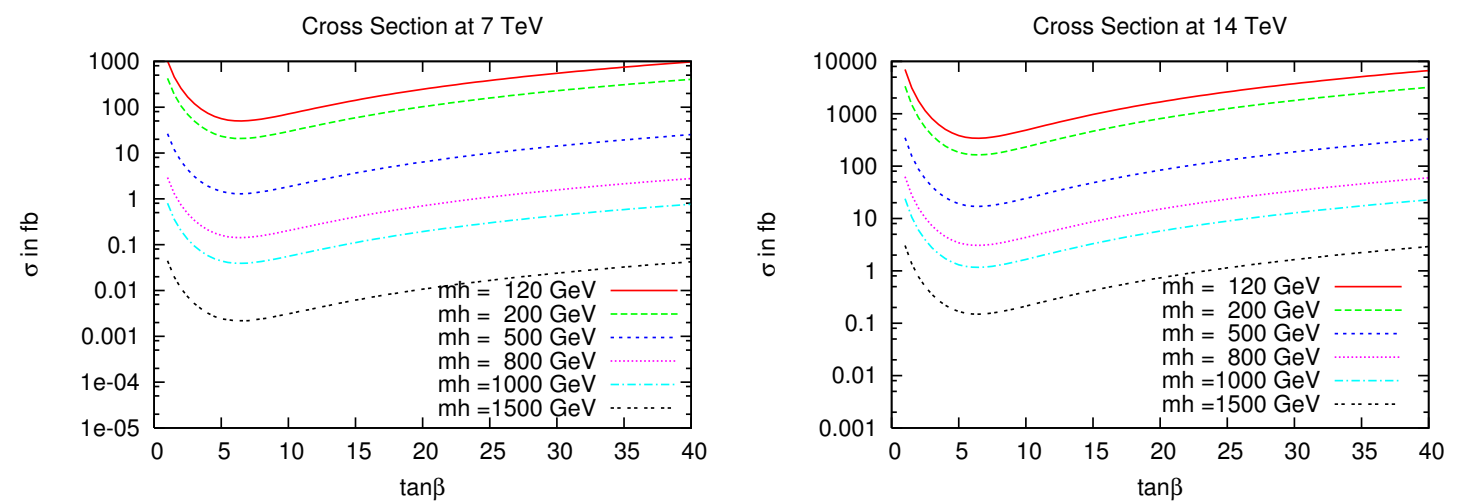

Figure 2. The cross section for top charged-Higgs production at LHC for two different cm energies, $7 \mathrm{TeV}$ (left) and $14 \mathrm{TeV}$ (right), as a function of $\tan \beta$ for various charged Higgs masses.

figure 2 for various values of charged Higgs masses. We show the cross section for two different center of mass energies of $7 \mathrm{TeV}$ and $14 \mathrm{TeV}$ for which the LHC is planned to operate and have used the leading order parton density function (PDF) sets of CTEQ6L1 [26]. We see that the cross sections have a similar profile for various $M_{H^{-}}$values and fall sharply for larger $M_{H^{-}}$. The cross sections are proportional to $\left(m_{t}^{2} \cot ^{2} \beta+m_{b}^{2} \tan ^{2} \beta\right)$, which is minimized for $\tan \beta=\sqrt{\frac{m_{t}}{m_{b}}} \simeq 6.41$, independent of the center-of-mass energy and the value of $M_{H^{-}}$. This can indeed be seen from figure 2 . Here we have taken the top mass to be $172.6 \mathrm{GeV}$ and have evaluated the PDF's at the same scale.

The $t b H^{-}$vertex has a scalar-pseudoscalar $\left(A+B \gamma^{5}\right)$ chiral structure which is different from vector-axial vector coupling of the $t b W$ and $t \bar{t} Z^{0}$ vertices. One thus expects a very different longitudinal polarization asymmetry given by eq. (2.4) for top charged-Higgs production compared to $t \bar{t}$ production, and for the closely related process of associated $t W$ production in the SM proceeding via $g b \rightarrow t W$. For SM $t W$ production we find the longitudinal polarization to be $P_{t} \simeq-0.25$; for $t \bar{t}$ production it is $\mathcal{O}\left(-10^{-4}\right)$. The very small value of $P_{t}$ for top pair production in the SM is because the dominant contribution for both $g g \rightarrow t \bar{t}$ and $q \bar{q} \rightarrow t \bar{t}$ comes from chirality conserving $s$-channel gluon exchange processes, resulting in the production of largely unpolarized tops. These values of $P_{t}$ have also been calculated in [27], where top polarization effects for top-slepton production in $R$-parity violating SUSY was considered. We show the polarization asymmetry for $t H^{-}$ production in figure 3 as a function of $\tan \beta$ for both $\sqrt{s}=7$ and $14 \mathrm{TeV}$. In contrast to the related case of top-slepton production considered in [27] where $P_{t}$ was found to be independent of the $R$-parity violating SUSY $t b \tilde{l}$ coupling, here $P_{t}$ does have an interesting dependence on $\tan \beta$. As mentioned previously, we notice the interesting feature that the polarization vanishes at $\tan \beta=\sqrt{\frac{m_{t}}{m_{b}}}$ for all $M_{H^{-}}$and $\hat{s}$, as expected from the vanishing of the chiral part of the coupling (3.2) at this $\tan \beta$ value, the same value for which the cross sections are minimized. The curves change sign at this point and saturate rapidly for larger $\tan \beta$ values.

A plot of $P_{t}$ vs the charged Higgs mass for various values of $\tan \beta$ is shown in figure 4 , 

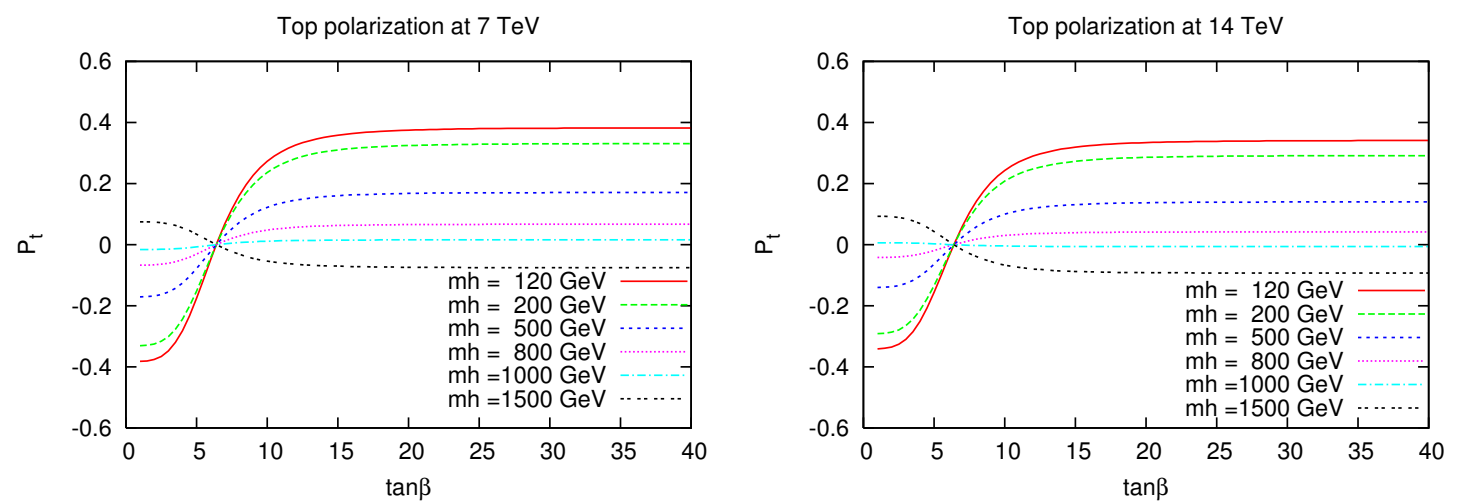

Figure 3. The polarization asymmetries for top charged-Higgs production at LHC for two different cm energies, $7 \mathrm{TeV}$ (left) and $14 \mathrm{TeV}$ (right), as a function of $\tan \beta$ for various charged Higgs masses.
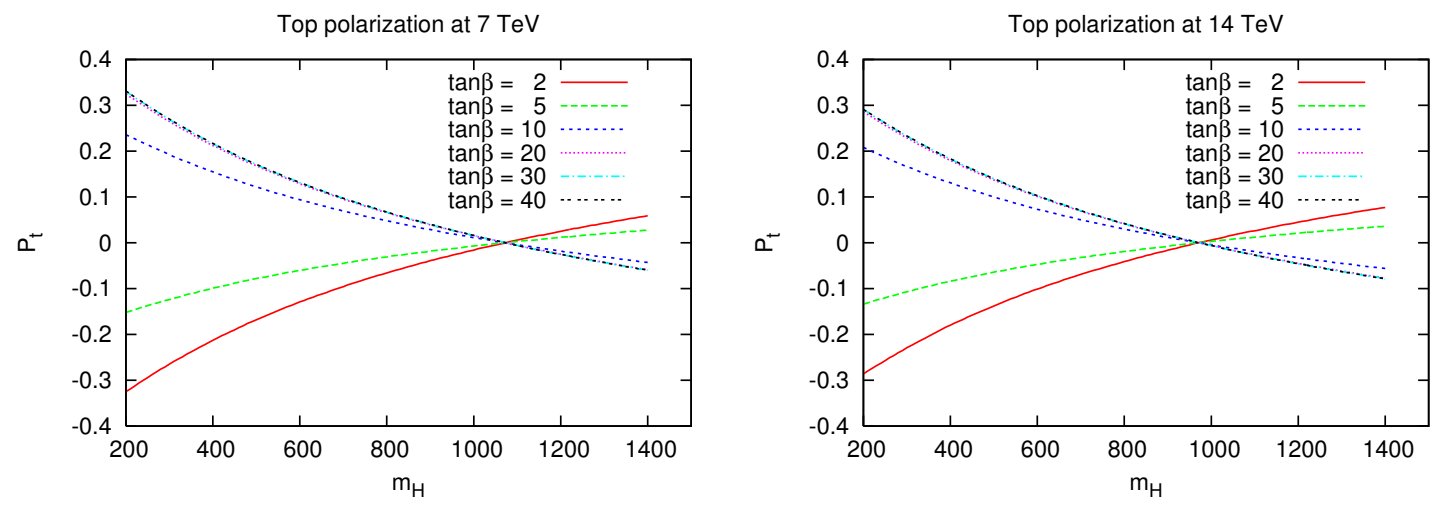

Figure 4. The polarization asymmetry for top charged-Higgs production at LHC for a cm energy of $7 \mathrm{TeV}$ (left) and $14 \mathrm{TeV}$ (right), as a function of $M_{H^{-}}$for various $\tan \beta$ values.

for $\sqrt{s}=7$ and $14 \mathrm{TeV}$. We notice that the polarization asymmetry vanishes for a charged Higgs mass close to $1100 \mathrm{GeV}$ for $\sqrt{s}=7 \mathrm{TeV}$ and around $1000 \mathrm{GeV}$ for the $14 \mathrm{TeV}$ case, for all $\tan \beta$, and changes sign as $M_{H^{-}}$is increased. This can be understood as follows. In the expression for the polarization asymmetry $P_{t} \propto \rho(+,+)-\rho(-,-)$, the angular integrals can be done analytically. Since the parton distributions of the gluon and $b$ quark peak at low $x$, the remaining PDF integrals over the momentum fractions of the gluon and $b$ are dominated at low $x$, i.e, at the threshold for top charged-Higgs production. One can show that the expressions for $P_{t}$, expanded in powers of the top momentum $p_{t}$ (i.e, evaluated close to $\left.\hat{s}=\left(m_{t}+M_{H^{-}}\right)^{2}\right)$, vanishes for $M_{H^{-}}=6 m_{t} \simeq 1035.6 \mathrm{GeV}$ at leading order in $p_{t}$, for all $\tan \beta$, in reasonable agreement with figure 4 . Of course, one cannot get an exact analytic expression for $M_{H^{-}}$when $P_{t}$ vanishes without doing the numerical integrals over the gluon and $b$ quark PDF's. Still, the above argument, which is independent of the centerof-mass energy of the colliding protons, is useful for understanding why the polarization vanishes close to $M_{H^{-}} \simeq 1000 \mathrm{GeV}$ for both $\sqrt{s}=7$ and $14 \mathrm{TeV}$.

The important point to note is that the magnitude and sign of these asymmetries are 
sensitively dependent on $M_{H^{-}}$and $\tan \beta$ values and are significantly different from the case of $t W$ and $t \bar{t}$ production, because of the different chiral structure of the $t b W$ vertex.

\section{Azimuthal distributions of decay leptons}

As mentioned in previous sections, the top quark decays rapidly and its properties have to be deduced from its decay products. The top polarization can be determined by the angular distribution of its decay products using eq. (2.1). The lab frame polar distribution of the lepton is independent of the anomalous $t b W$ decay vertex. However, we find that it is not sensitive to model parameters and is largely indistinguishable from the $t W$ case in the SM.

As shown in [25] and references therein, the azimuthal angle of the decay lepton in the lab frame is sensitive to the top polarization and independent of possible new physics in the $t b W$ decay vertex and is thus a convenient probe. The lepton azimuthal angle $\phi_{\ell}$ is defined with respect to the top production plane chosen as the $x-z$ plane, with the beam direction as the $z$ axis and the convention that the $x$ component of the top momentum is positive. Since at the LHC, one cannot uniquely define a positive direction of $z$ axis, the lepton azimuthal distribution is identical for $\phi_{l}$ and $2 \pi-\phi_{l}$ and is symmetric around $\phi_{l}=\pi$.

The $\phi_{\ell}$ distributions for pure, i.e, $100 \%$, positively or negatively polarized top quark ensemble is obtained by using only the $(+,+)$ or $(-,-)$ density matrix elements respectively in eq. (2.7). This is, of course, expected to be different from that for an ensemble with a partial degree of polarization $P_{t}$. In computing the $\phi_{\ell}$ distributions we have taken into account the full spin coherence effects of the top encoded in the diagonal and off-diagonal elements of the production and decay spin density matrices.

With this choice of frame, the normalized lepton azimuthal distributions for $\sqrt{s}=$ $7 \mathrm{TeV}$ is shown in figure 5 for small and large values of $\tan \beta$, for various $M_{H^{-}}$values. The corresponding plots for a cm energy of $14 \mathrm{TeV}$ is shown in figure 6 . The $\phi_{\ell}$ distribution for $t W^{-}$production in the $\mathrm{SM}$ is also shown for comparison.

The $\phi_{\ell}$ distributions for other values of $\tan \beta$ and $M_{H^{-}}$have a similar profile, with a peak at $\phi_{\ell}=0$ and $2 \pi$. The $\phi_{\ell}$ distribution depends on both kinematic and top polarization effects and the factors which influence its shape can be understood as follows. According to eq. (2.1), the decay lepton is emitted preferentially along the top spin direction in the top rest frame, with $\kappa_{f}=1$. To obtain the distribution in the laboratory frame we use the following relation between the angle $\theta_{\ell}^{*}$ between the top spin and decay lepton in the top rest frame and the angle $\theta_{t \ell}$ between the top and lepton in the laboratory frame:

$$
\cos \theta_{\ell}^{*}=\frac{\cos \theta_{t \ell}-\beta}{1-\beta \cos \theta_{t \ell}}
$$

where

$$
\cos \theta_{t \ell}=\cos \theta_{t} \cos \theta_{\ell}+\sin \theta_{t} \sin \theta_{\ell} \cos \phi_{\ell}
$$



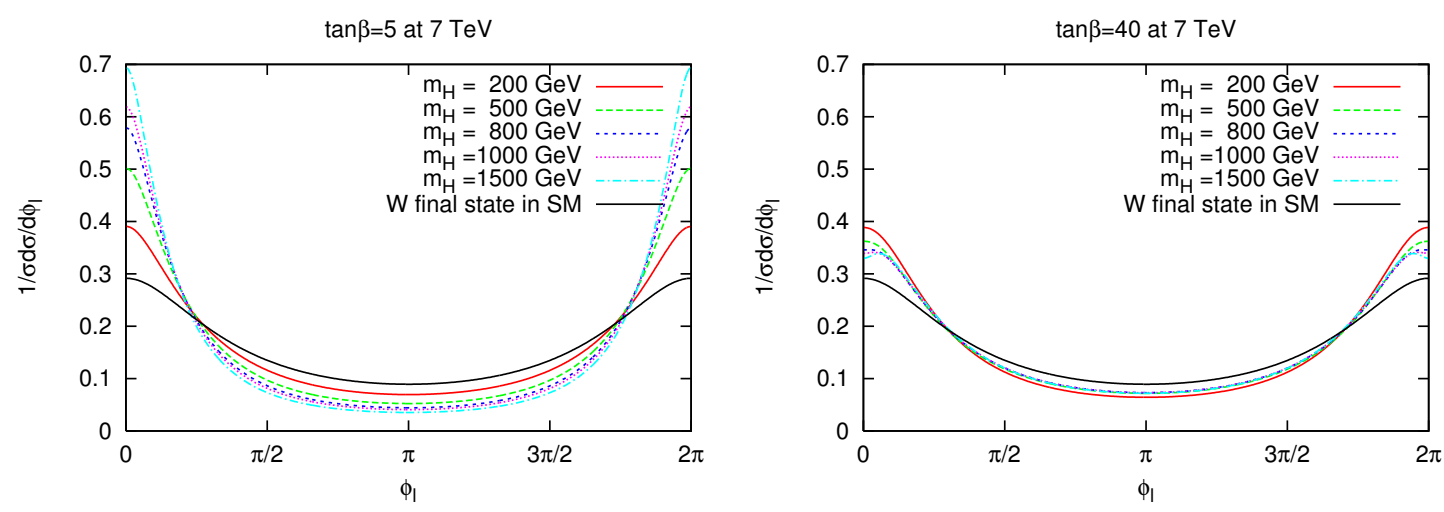

Figure 5. The normalized lepton azimuthal distribution for $\tan \beta=5$ (left) and $\tan \beta=40$ (right) for various charged Higgs masses at a cm energy of $7 \mathrm{TeV}$.
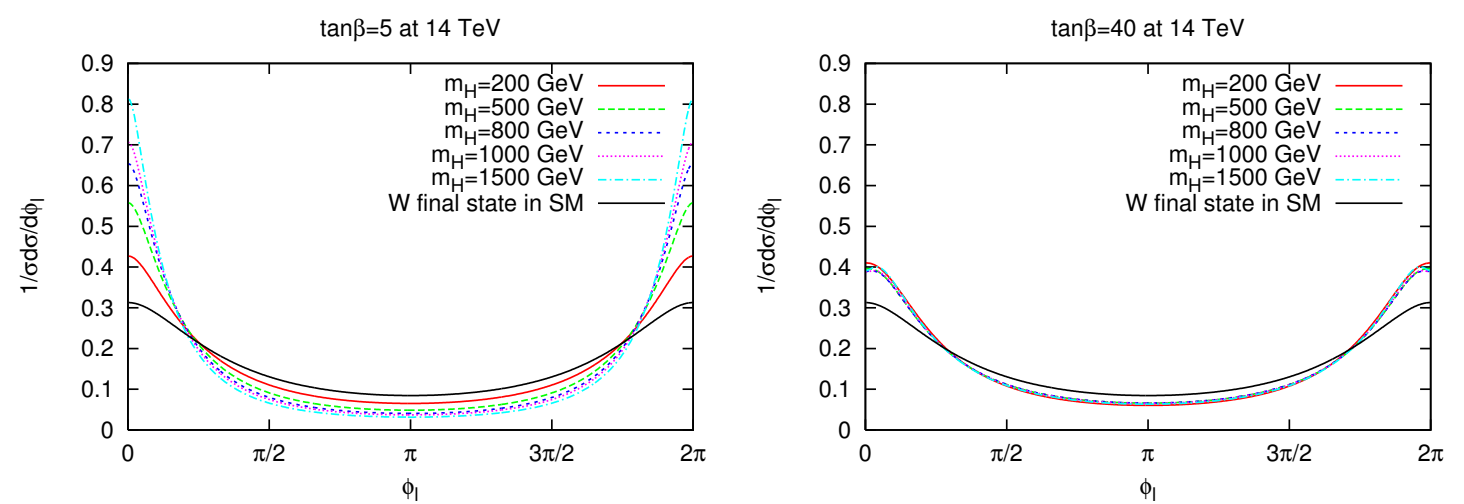

Figure 6. The normalized lepton azimuthal distribution for $\tan \beta=5$ (left) and $\tan \beta=40$ (right) for various charged Higgs masses at a cm energy of $14 \mathrm{TeV}$.

Using the above relations, the laboratory frame angular distribution of the lepton becomes

$$
\frac{1}{\Gamma_{\ell}} \frac{d \Gamma_{\ell}}{d \cos \theta_{t \ell}}=\frac{1}{2}\left(1-\beta^{2}\right)\left(1-P_{t} \beta\right) \frac{1+\frac{P_{t}-\beta}{1-P_{t} \beta} \cos \theta_{t \ell}}{\left(1-\beta \cos \theta_{t \ell}\right)^{3}},
$$

where $\beta=\sqrt{1-m_{t}^{2} / E_{t}^{2}}$ is the top velocity in the parton $\mathrm{cm}$ frame. We notice that the distribution (4.3) peaks for large $\cos \theta_{t \ell}$, since it occurs in the denominator and hence from eq. (4.2) for small $\phi_{\ell}$. Thus, the boost to the laboratory frame produces a collimating effect along the direction of the top momentum, which gets translated to a peaking at $\phi_{\ell}=0$.

We notice that the curves are separated at the peaks for different $M_{H^{-}}$values and are very different from the $t W$ case in the SM. As in [14, 25, 28, 29], we can quantify this difference by defining a normalized azimuthal asymmetry for the lepton as

$$
A_{\phi}=\frac{\sigma\left(\cos \phi_{\ell}>0\right)-\sigma\left(\cos \phi_{\ell}<0\right)}{\sigma\left(\cos \phi_{\ell}>0\right)+\sigma\left(\cos \phi_{\ell}<0\right)}
$$

where the denominator is the total cross section. A plot for $A_{\phi}$ as a function of $\tan \beta$ with and without cuts on the lepton momenta are shown in figure 7 for a $\mathrm{cm}$ energy of 

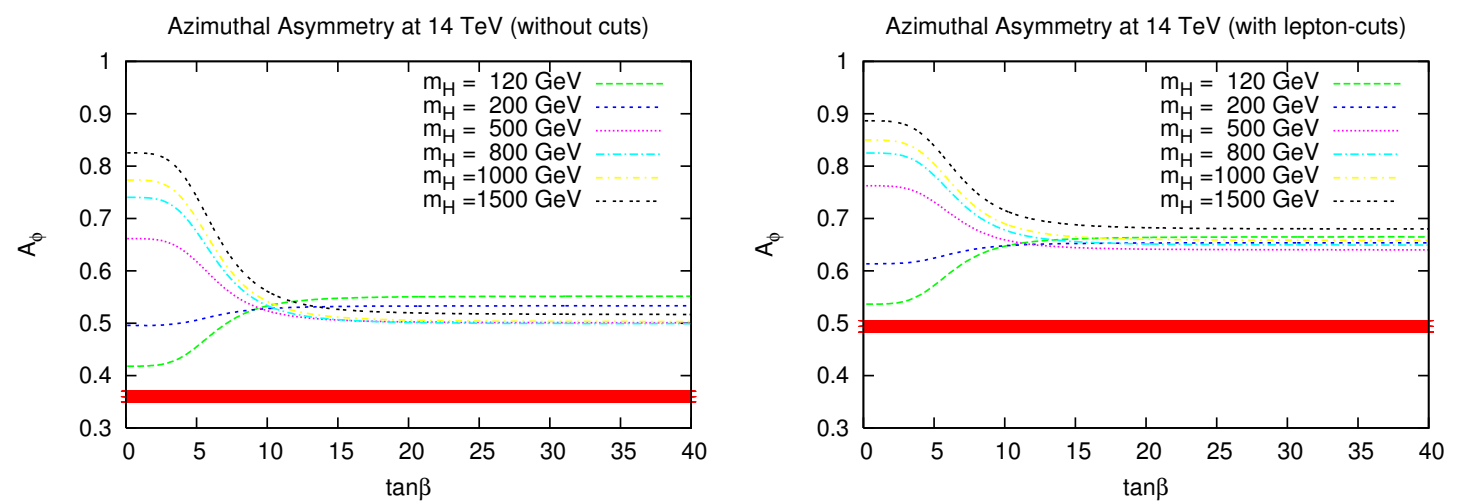

Figure 7. $A_{\phi}$ as a function of $\tan \beta$ and different charged Higgs masses at $\sqrt{s}=14 \mathrm{TeV}$ without lepton cuts (left) and with cuts (right). The red band corresponds to the azimuthal asymmetry for $t W$ production in the SM with a $2 \sigma$ error interval.
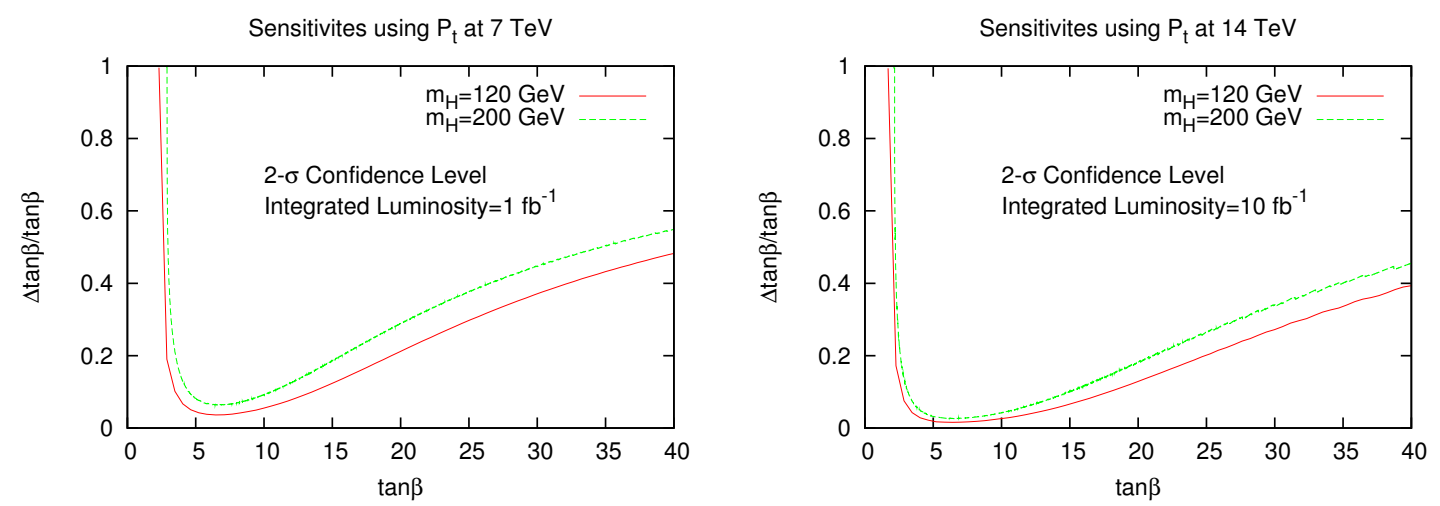

Figure 8. The fractional accuracy of $\tan \beta$ at $2 \sigma$ CL as a function of $\tan \beta$ for $\sqrt{s}=7 \mathrm{TeV}$ (left) and $14 \mathrm{TeV}$ (right) using the polarization $P_{t}$, with $\int \mathcal{L} d t=1 \mathrm{fb}^{-1}$ and $10 \mathrm{fb}^{-1}$ respectively.

$14 \mathrm{TeV}$. We have used the following rapidity and transverse momentum acceptance cuts on the decay lepton: $|\eta|<2.5, p_{T}^{\ell}>20 \mathrm{GeV}$. Also shown is the SM value for $A_{\phi}$ for $t W$ production with a $2 \sigma$ error band.

The lepton cuts only mildly increase the value of $A_{\phi}$ for the charged Higgs case and the value for $t W$ production in the SM is also enhanced from about 0.35 without cuts to about 0.5 with cuts, as can been seen from figure 7 . The azimuthal asymmetry also shows considerable variation, as a function of $\tan \beta$, roughly in the range $3 \lesssim \tan \beta \lesssim 15$ and becomes flat for values outside this range and almost independent of $M_{H^{-}}$. From figure 3 , we see that this is the same range of $\tan \beta$ for which the polarization $P_{t}$ shows variation, becoming constant for roughly $\tan \beta>15$; thus, the azimuthal asymmetry follows the same trends as the top polarization. If the mass of the charged Higgs is known, from a measurement of $A_{\phi}$ it would be easier to determine $\tan \beta$ if it lies within this range.

We now investigate the accuracy to which one can determine $\tan \beta$ from the top polarization, $P_{t}$, and the azimuthal asymmetry, $A_{\phi}$. The accuracy of the determination of 

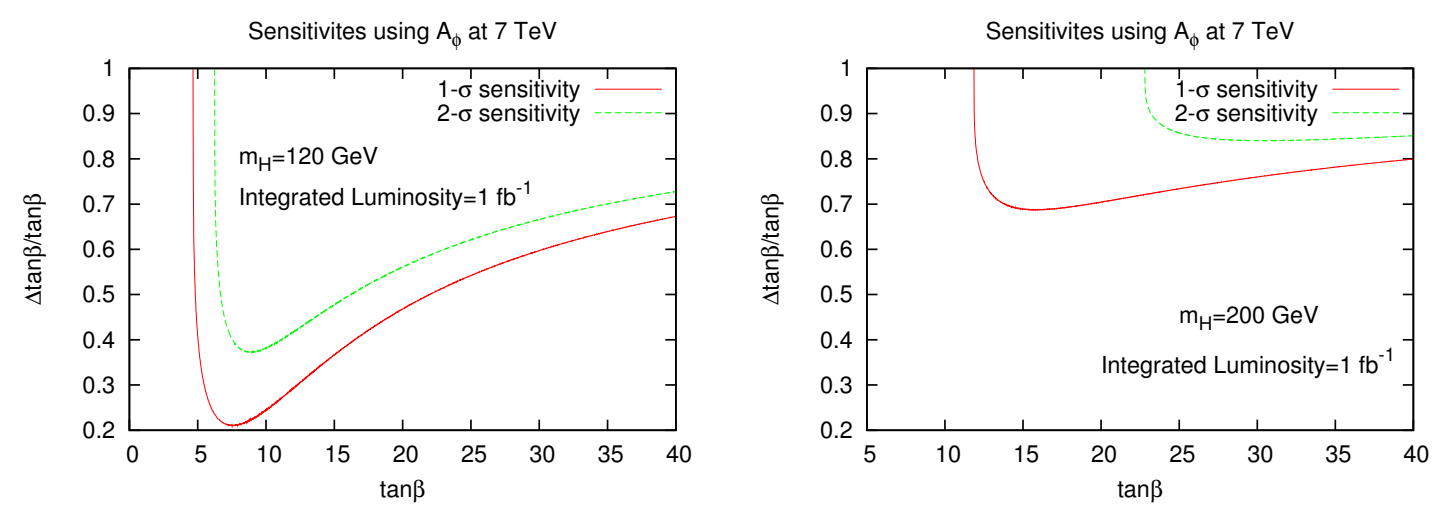

Figure 9. The fractional accuracy of $\tan \beta$ as a function of $\tan \beta$ for $\sqrt{s}=7 \mathrm{TeV}$ using the azimuthal asymmetry $A_{\phi}$ for $M_{H^{-}}=120 \mathrm{GeV}$ (left) and $M_{H^{-}}=200 \mathrm{GeV}$ (right).

parameter $\tan \beta$ at $\tan \beta_{0}$, from the measurement of an observable $O(\tan \beta)$, is $\Delta \tan \beta$ if $\left|O(\tan \beta)-O\left(\tan \beta_{0}\right)\right|<\Delta O\left(\tan \beta_{0}\right)$ for $\left|\tan \beta_{0}-\tan \beta\right|<\Delta \tan \beta$, where $\Delta O\left(\tan \beta_{0}\right)$ is the statistical fluctuation in $O$ at an integrated luminosity $\mathcal{L}$. The corresponding fractional accuracy is then $\Delta \tan \beta / \tan \beta_{0}$. For top-polarization, $P_{t}$ and azimuthal asymmetry, $A_{\phi}$, the statistical fluctuations at a level of confidence $f$ are given by $\Delta O=f / \sqrt{\mathcal{L} \sigma} \times \sqrt{1-O^{2}}$, where $O$ denotes $P_{t}$ or $A_{\phi}$.

In figure 8 , we show the fractional accuracy $\Delta \tan \beta / \tan \beta$ in the determination of the coupling $\tan \beta$ from the polarization $P_{t}$ at $2 \sigma$ confidence level (CL). We choose, for illustration, charged Higgs masses of 120 and $200 \mathrm{GeV}$ and an integrated luminosity of $1 \mathrm{fb}^{-1}$ and $10 \mathrm{fb}^{-1}$ for $\sqrt{s}=7$ and $14 \mathrm{TeV}$ respectively. We use, for convenience, the criterion $\Delta \tan \beta / \tan \beta<0.3$ for an accurate determination of $\tan \beta$ since this corresponds to a relative accuracy of about $1 \%$ in the determination of physical quantities, which are proportional to the square of the couplings.

Then, we see that at $\sqrt{s}=7 \mathrm{TeV}, \tan \beta$ can be determined accurately for values between roughly 3 and 25 for $M_{H^{-}}=120 \mathrm{GeV}$ and between 3 and 20 for $M_{H^{-}}=200 \mathrm{GeV}$. The corresponding range for $\tan \beta$ determination for the LHC running at $14 \mathrm{TeV}$ are 3 to 30 for $M_{H^{-}}=120 \mathrm{GeV}$ and 3 to 25 for $M_{H^{-}}=200 \mathrm{GeV}$. For larger $\tan \beta$ (and even for very low $\tan \beta$ ) the sensitivity worsens since the $P_{t}$ curves become flat and do not show much variation as a function of $\tan \beta$, as can be seen from figure 3 . One can, of course, choose a different value for $\Delta \tan \beta / \tan \beta$ as a measure of $\tan \beta$ accuracy in which case the corresponding limits on $\tan \beta$ will be different as can be read from the plots.

We now consider the accuracy to which $\tan \beta$ can be determined from the more conveniently measurable azimuthal asymmetry. Plots of the fractional accuracy for this case are shown in figure 9 and figure 10 for the cases of $\sqrt{s}=7 \mathrm{TeV}$ and $14 \mathrm{TeV}$ respectively and with the indicated charged Higgs masses and luminosities. If we use the same criterion for $\tan \beta$ accuracy as before, $\Delta \tan \beta / \tan \beta<0.3$, we notice that for a cm energy of $7 \mathrm{TeV}$ and an integrated luminosity of $1 \mathrm{fb}^{-1}$, the azimuthal asymmetry is not a very sensitive measure of $\tan \beta$. For the lower charged Higgs mass of $120 \mathrm{GeV}$, and at a $1 \sigma \mathrm{CL}, \tan \beta$ 

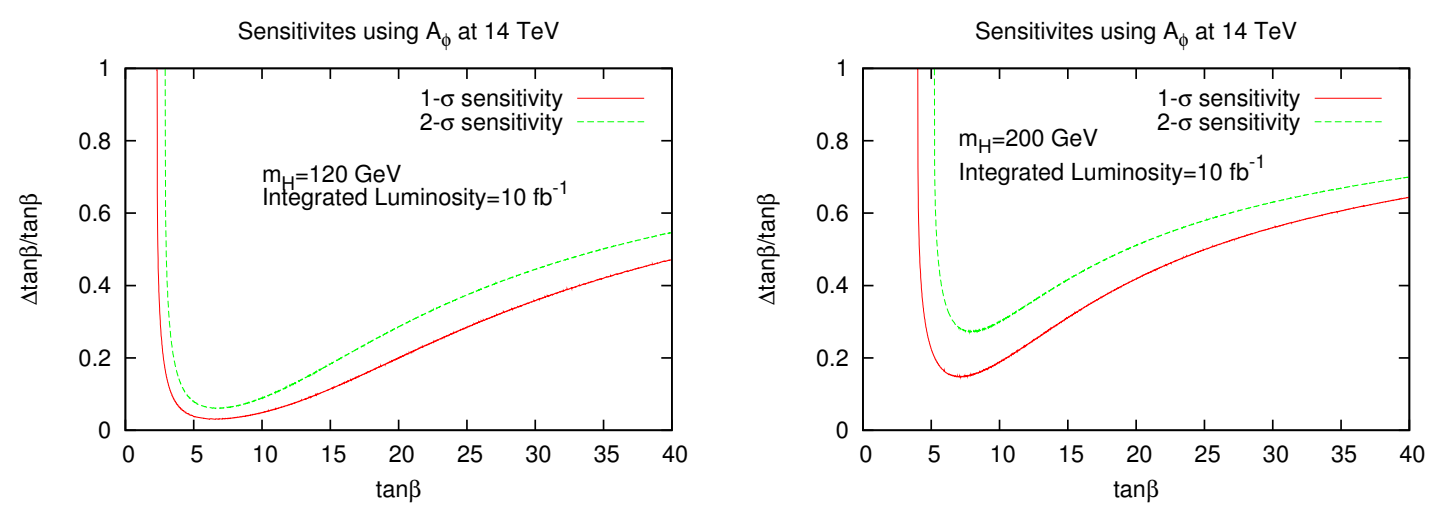

Figure 10. The fractional accuracy of $\tan \beta$ as a function of $\tan \beta$ for $\sqrt{s}=14 \mathrm{TeV}$ using the azimuthal asymmetry $A_{\phi}$ for $M_{H^{-}}=120 \mathrm{GeV}$ (left) and $M_{H^{-}}=200 \mathrm{GeV}$ (right).

can be probed roughly in the range 6 to 12; the sensitivity worsens for larger charged Higgs masses or CL's. The top polarization is better probe of $\tan \beta$ than the azimuthal asymmetry. However, this is due to the fact that in constructing the asymmetry only the semi-leptonic decay modes of the top have been considered, which reduces the cross section by a factor of 3 . The sensitivities are considerably enhanced if we include all decay channels of the top. But it must be remembered that using any decay product of the top other than $\ell^{+}$and $\bar{d}$ to construct the azimuthal asymmetry will make $A_{\phi}$ dependent on new physics in the $t b W$ vertex. For the LHC running at $\sqrt{s}=14 \mathrm{TeV}, A_{\phi}$ is a more sensitive measure of $\tan \beta$ compared to the $7 \mathrm{TeV}$ case, at least for the lower charged Higgs mass of $120 \mathrm{GeV}$. For this case $\tan \beta$ can be probed in the range 3 to 25 at the $1 \sigma$ CL and between 3 and 20 at a $2 \sigma \mathrm{CL}$. For $M_{H^{-}}=200 \mathrm{GeV}, A_{\phi}$ is sensitive to $\tan \beta$ only at the $1 \sigma \mathrm{CL}$ for a smaller range of 5 to 15 .

As is to be expected, $\tan \beta$ can be determined to a higher accuracy and for a larger range using the top polarization $P_{t}$, compared to the azimuthal asymmetry constructed from the decay lepton; the restriction to semi-leptonic decay modes of the top further reduces the sensitivity to $A_{\phi}$. However, it is interesting to note that the profile of the plot of $\Delta \tan \beta / \tan \beta$ vs $\tan \beta$ computed by using $A_{\phi}$, shown in figure 9 and 10 , is similar to that obtained by using the polarization $P_{t}$, shown in figure $8 . A_{\phi}$ follows the change in $P_{t}$ as a function of the coupling $\tan \beta$ and is thus a faithful probe of the top polarization itself. At least for $\sqrt{s}=14 \mathrm{TeV}$ and $M_{H^{-}}=120 \mathrm{GeV}$, the range in which $\tan \beta$ can be probed accurately using $A_{\phi}$ or $P_{t}$ is roughly similar for both variables.

Thus, the azimuthal asymmetry can be a convenient and sensitive probe of both the top polarization and the coupling parameter $\tan \beta$ in the THDM, at least in the regions of parameter space mentioned above.

It is worthwhile to comment on the dominant backgrounds to our signal process $g b \rightarrow$ $t H^{-} \rightarrow t \bar{t} b$. When $M_{H^{-}}>m_{t}+m_{b}$, we require the top to decay semi-leptonically and the anti-top to decay hadronically to trigger on the charged Higgs signal, as well as for the purpose of reconstruction of the top quarks and the charged Higgs. The complete final 
state therefore consists of $3 b$ jets +2 light jets +1 lepton + missing energy. The main background for this signal would come from next-to-leading order NLO QCD processes, which are (a) $g g \rightarrow t \bar{t} b \bar{b}$, (b) $g b \rightarrow t \bar{t} b$, and (c) $g g \rightarrow t \bar{t} g$, where in the first case, one of the $\mathrm{b}$ jets is missed and in the last case the gluon jet is mis-tagged as a $b$ jet (with probability of around $1 \%$ ). Refs. [30-32] have investigated the charged-Higgs signal in this process in great detail for the LHC with triple $b$-tagging. They have used kinematical cuts of $p_{T}>30 \mathrm{GeV}$ and $|\eta|<2.5$ for all jets and assume $b$-tagging efficiency of $40 \%$ in their analysis. The conclusion from their analysis for $30 \mathrm{fb}^{-1}$ of accumulated data is that there are enough number of events for charged Higgs discovery in this channel at the 5- $\sigma$ level upto a mass of $600 \mathrm{GeV}$ for very large values of $\tan \beta(>25)$ and very small values of $\tan \beta$ $(<5)$. We can expect better visibility for the charged Higgs when the $b$-tagging efficiency increases in future. Backgrounds from weak processes like $t W+X, b \bar{b}+X$ and $W+2 j$ would be suppressed because we choose the signal to consist of $3 b$ jets and an isolated lepton.

When $M_{H^{-}}<m_{t}+m_{b}$, the dominant decay of the $H^{-}$is into $\tau+\bar{\nu}_{\tau}$. Our signal in this will be $g b \rightarrow t H^{-} \rightarrow t \tau^{-} \bar{\nu}_{\tau} \rightarrow b \ell^{+} \nu_{\ell} \tau^{-} \bar{\nu}_{\tau}$. For this final state of $b+$ lepton $+\tau+$ missing energy, the background now comes from the processes of $t \bar{t}$ production with the $\bar{t}$ decaying into a $\tau$ and $t W^{-}$production with $W^{-}$decaying into a $\tau$. In both these cases, since the $\tau$ comes from $W^{-}$decay, $\tau$ polarization can be used to suppress the background [33, 34]. While the presence of two neutrinos in the final state would seem to make it impossible to reconstruct the top production plane needed for our analysis, we are helped by the fact that the $t H^{-}$events are produced close to the threshold because of the sharp peaking of the initial-state partons at low $x$. Thus it is a reasonable approximation to treat the top quark and the charged Higgs as at rest, enabling approximate determination of the energy and momenta of both neutrinos on an event-by-event basis.

The NLO QCD corrections to the process $g b \rightarrow t H^{-}$have been studied in refs. [35, 36] and next-to-next-to-leading-order (NNLO) soft gluon corrections have been evaluated in ref. [37]. These corrections are shown to be substantial, upto $85 \%$ of the LO cross section for large Higgs masses. It has been also shown that the K-factor in this process is proportional to the mass of charged Higgs. Since QCD corrections are model independent, one can use the K-factor appropriately in the analysis to rescale the LO result to the NLO order. The normalized differential cross sections and the asymmetries we calculate would be insensitive to the higher order corrections. We have not used any K-factor in our analysis. Including NLO QCD corrections through the naive use of K-factor would increase our signal cross section by a factor of 1.5-1.85 depending upon the charged Higgs mass and hence sensitivity to the parameters would increase.

The complete NLO EW calculations for the process $g b \rightarrow t H^{-}$have been done in ref. [38] for type II 2HDM. They have reported that the NLO EW correction to the total cross section is very mild. It varies from less than $1 \%$ for low values of $\tan \beta$ to less than $4 \%$ for higher values of $\tan \beta$. The effects of NLO EW corrections to observables like top polarization, normalized angular distributions and angular asymmetries are expected to be small. For example, in ref. [15], it has been shown that NLO EW supersymmetric effects on top polarization is almost zero for all values of charged Higgs masses and all values of $\tan \beta$ except for $\tan \beta \approx 10$, for which correction is around $-1 \%$ to $-3 \%$. 
Any NLO corrections to top decay will not affect our analysis of charged lepton angular distributions and asymmetries as it has been proven that charged lepton angular distributions are independent of any corrections to form factors in top decay. There can also be NLO corrections from non-factorizable diagrams. However, this analysis has not been done in the literature so far and it would be interesting to see the effect of these non-factorizable diagrams to our analysis which is beyond the scope of this work.

\section{Summary}

We have studied the issue of using the polarization of the top quark produced in association with a charged Higgs in the type II THDM or SUSY models as a probe of the coupling parameter $\tan \beta$ occurring in such models. Since the top decays before it has the time to hadronize, its polarization, reflected in the angular distribution of its decay products, can be a probe of new physics underlying its production. We have derived analytic expressions for left and right polarized $t H^{-}$production (and the off-diagonal elements as well in the spin density matrix). Essentially because of the scalar-pseudoscalar coupling (3.2) of the $t b H^{-}$vertex, compared to the vector-axial vector couplings of the top in the SM, the resulting polarizations are vastly different from that expected in the SM and are sensitively dependent on the charged Higgs mass and $\tan \beta$, as shown in figures 3 and 4 , where we considered both the cm energies of 7 and $14 \mathrm{TeV}$ at which the LHC is planned to run. The degree of longitudinal top polarization can be as large as 0.3 to 0.4 (for a charged Higgs mass of $120 \mathrm{GeV}$ and for $\tan \beta$ values less than 5 and greater than 10), compared to the SM values of -0.25 for $t W$ production or $\mathcal{O}\left(-10^{-4}\right)$ for $t \bar{t}$ production. Characteristic of the $t b H^{-}$coupling in the THDM, the $2 \rightarrow 2$ top production cross sections are minimized and the polarizations vanish and change sign as a function of $\tan \beta$ at $\tan \beta=\sqrt{\frac{m_{t}}{m_{b}}}$.

We then investigated to what extent top polarization is reflected in the angular distribution of the decay lepton in the process $t \rightarrow b W^{+} \rightarrow b \nu_{\ell} \ell^{+}$, with inclusive decay of the $b$ and $H^{-}$. Since it is known that the laboratory frame angular distributions of the charged lepton in top decay depends only on the top production process and are independent of new physics in the $t b W$ vertex, we considered the azimuthal distribution of the lepton from top decay, $A_{\phi}$, as a probe of new physics in its production (we find the polar distribution of the lepton in the THDM insensitive to $\tan \beta$ and the charged Higgs mass and almost identical to $t W$ production in the $\mathrm{SM})$. $A_{\phi}$ is sensitive to $\tan \beta$ values roughly in the range $3 \lesssim \tan \beta \lesssim 15$, for different charged Higgs masses considered and becomes constant for larger $\tan \beta$ values. This is the same range in which the top polarization shows variation as a function of $\tan \beta ; A_{\phi}$ thus captures the dependence of $P_{t}$ on $\tan \beta$. If the charged Higgs mass is already known, a measurement of $A_{\phi}$ can help measure $\tan \beta$ if it lies in the above range.

We also computed the fractional accuracy to which $\tan \beta$ can be measured, as a function of $\tan \beta$, from the top polarization $P_{t}$ and a measurement of the azimuthal asymmetry $A_{\phi}$. Using the criterion that $\Delta \tan \beta / \tan \beta<0.3$ for an accurate determination of $\tan \beta$, we find that $P_{t}$ can help determine $\tan \beta$ lying in the range between 3 and 25 for a cm energy of $7 \mathrm{TeV}$ and between 3 and 30 for the $14 \mathrm{TeV}$ case, at a $2 \sigma \mathrm{CL}$ for $M_{H^{-}}=120 \mathrm{GeV}$; the range is only slightly smaller for a larger $M_{H^{-}}$of $200 \mathrm{GeV}$. While the azimuthal asymmetry 
is not very sensitive to an accurate measurement of $\tan \beta$ for the $\mathrm{LHC}$ running at $7 \mathrm{TeV}$, we find that at $14 \mathrm{TeV}$ one can use the azimuthal asymmetry to probe $\tan \beta$ up to 25 at a $1 \sigma \mathrm{CL}$ and for $M_{H^{-}}=120 \mathrm{GeV}$; for $M_{H^{-}}=200 \mathrm{GeV}$ the corresponding range is 5 to 15. Including both leptonic and hadronic decay modes of the top is expected to increase the sensitivity of the azimuthal asymmetry to $\tan \beta$; however, this renders the asymmetry sensitive to new physics in the $t b W$ decay vertex, apart from new physics in top production.

The sensitivity plot for $\tan \beta$ determination using $A_{\phi}$ follows roughly the one obtained by using $P_{t}$. Thus, the azimuthal asymmetry of the decay lepton can be a convenient and accurate probe of the top polarization and the coupling parameter $\tan \beta$ of the THDM or SUSY models for the LHC running at $\sqrt{s}=14 \mathrm{TeV}$ and for smaller charged Higgs masses.

\section{Acknowledgments}

K.H and K.R gratefully acknowledge support from the Academy of Finland (Project No. 115032). S.K.R is supported by US Department of Energy, Grant Number DE-FG0204ER41306. S.D.R. thanks Helsinki Institute of Physics and the University of Helsinki for hospitality during the period when this work was completed.

\section{References}

[1] J.F. Gunion, H.E. Haber, G.L. Kane and S. Dawson, The Higgs Hunters Guide, Westview Press, Boulder, CO (2000).

[2] G.C. Branco, L. Lavoura and J.P. Silva, CP Violation, Int. Ser. Monogr. Phys. 103 (1999) 1.

[3] A. Djouadi, The Anatomy of electro-weak symmetry breaking. II. The Higgs bosons in the minimal supersymmetric model, Phys. Rept. 459 (2008) 1 [hep-ph/0503173] [SPIRES].

[4] M.S. Carena and H.E. Haber, Higgs boson theory and phenomenology, Prog. Part. Nucl. Phys. 50 (2003) 63 [hep-ph/0208209] [SPIRES].

[5] R.N. Mohapatra and G. Senjanović, Neutrino mass and spontaneous parity nonconservation, Phys. Rev. Lett. 44 (1980) 912 [SPIRES].

[6] R.N. Mohapatra and G. Senjanović, Neutrino Masses and Mixings in Gauge Models with Spontaneous Parity Violation, Phys. Rev. D 23 (1981) 165 [SPIRES].

[7] M. Magg and C. Wetterich, Neutrino mass problem and gauge hierarchy, Phys. Lett. B 94 (1980) 61 [SPIRES].

[8] G. Lazarides, Q. Shafi and C. Wetterich, Proton Lifetime and Fermion Masses in an SO(10) Model, Nucl. Phys. B 181 (1981) 287 [SPIRES].

[9] J. Schechter and J.W.F. Valle, Neutrino Masses in $\mathrm{SU}(2) \times \mathrm{U}(1)$ Theories, Phys. Rev. D 22 (1980) 2227 [SPIRES].

[10] I.I.Y. Bigi, Y.L. Dokshitzer, V.A. Khoze, J.H. Kuhn and P.M. Zerwas, Production and Decay Properties of Ultraheavy Quarks, Phys. Lett. B 181 (1986) 157 [SPIRES].

[11] W. Bernreuther, Top quark physics at the LHC, J. Phys. G 35 (2008) 083001 [arXiv: 0805.1333] [SPIRES].

[12] M. Beneke et al., Top quark physics, hep-ph/0003033 [SPIRES]. 
[13] W. Wagner, Top quark physics in hadron collisions, Rept. Prog. Phys. 68 (2005) 2409 [hep-ph/0507207] [SPIRES].

[14] R.M. Godbole, K. Rao, S.D. Rindani and R.K. Singh, On measurement of top polarization as a probe of $t \bar{t}$ production mechanisms at the LHC, JHEP 11 (2010) 144 [arXiv:1010.1458] [SPIRES].

[15] M. Beccaria, F.M. Renard and C. Verzegnassi, Electroweak supersymmetric effects on high energy unpolarized and polarized single top production at $\mathrm{LHC}$,

Phys. Rev. D 71 (2005) 033005 [hep-ph/0410089] [SPIRES].

[16] D. Espriu and J. Manzano, A study of top polarization in single-top production at the LHC, Phys. Rev. D 66 (2002) 114009 [hep-ph/0209030] [SPIRES].

[17] D. Espriu and J. Manzano, Single top production at the LHC: The Effective $W$ approximation, hep-ph/0109059 [SPIRES].

[18] D. Espriu and J. Manzano, Measuring effective electroweak couplings in single top production at the LHC, Phys. Rev. D 65 (2002) 073005 [hep-ph/0107112] [SPIRES].

[19] G. Mahlon and S.J. Parke, Angular correlations in top quark pair production and decay at hadron colliders, Phys. Rev. D 53 (1996) 4886 [hep-ph/9512264] [SPIRES].

[20] G. Mahlon and S.J. Parke, Maximizing spin correlations in top quark pair production at the Tevatron, Phys. Lett. B 411 (1997) 173 [hep-ph/9706304] [SPIRES].

[21] T. Stelzer and S. Willenbrock, Spin correlation in top quark production at hadron colliders, Phys. Lett. B 374 (1996) 169 [hep-ph/9512292] [SPIRES].

[22] W. Bernreuther, A. Brandenburg, Z.G. Si and P. Uwer, Top quark spin correlations at hadron colliders: Predictions at next-to-leading order QCD,

Phys. Rev. Lett. 87 (2001) 242002 [hep-ph/0107086] [SPIRES].

[23] W. Bernreuther, A. Brandenburg, Z.G. Si and P. Uwer, Top quark pair production and decay at hadron colliders, Nucl. Phys. B 690 (2004) 81 [hep-ph/0403035] [SPIRES].

[24] M. Arai, N. Okada, K. Smolek and V. Simak, Top quark spin correlations in the Randall-Sundrum model at the CERN Large Hadron Collider, Phys. Rev. D 75 (2007) 095008 [hep-ph/0701155] [SPIRES].

[25] R.M. Godbole, S.D. Rindani and R.K. Singh, Lepton distribution as a probe of new physics in production and decay of the $t$ quark and its polarization, JHEP 12 (2006) 021 [hep-ph/0605100] [SPIRES].

[26] J. Pumplin, A. Belyaev, J. Huston, D. Stump and W.K. Tung, Parton distributions and the strong coupling: CTEQ6AB PDFs, JHEP 02 (2006) 032 [hep-ph/0512167] [SPIRES].

[27] M. Arai, K. Huitu, S.K. Rai and K. Rao, Single production of sleptons with polarized tops at the Large Hadron Collider, JHEP 08 (2010) 082 [arXiv:1003.4708] [SPIRES].

[28] B.C. Allanach et al., Les Houches 'Physics at TeV colliders 2005' Beyond the standard model working group: Summary report, hep-ph/0602198 [SPIRES].

[29] R.M. Godbole, S.D. Rindani, K. Rao and R.K. Singh, Top polarization as a probe of new physics, AIP Conf. Proc. 1200 (2010) 682 [arXiv:0911.3622] [SPIRES].

[30] S. Moretti and D.P. Roy, Detecting heavy charged Higgs bosons at the LHC with triple b-tagging, Phys. Lett. B 470 (1999) 209 [hep-ph/9909435] [SPIRES]. 
[31] S. Lowette, J. Heyninck and P. Vanlaer, Heavy Charged MSSM Higgs Bosons in the $H^{ \pm} \rightarrow t b$ Decay in CMS, Report No. CMS CR 2004/031.

[32] K.A. Assamagan and N. Gollub, The ATLAS discovery potential for a heavy charged Higgs boson in $g g \rightarrow t b H^{ \pm}$with $H^{ \pm} \rightarrow t b$, Eur. Phys. J. C 39S2 (2005) 25 [hep-ph/0406013] [SPIRES].

[33] D.P. Roy, Looking for the charged Higgs boson, Mod. Phys. Lett. A 19 (2004) 1813 [hep-ph/0406102] [SPIRES].

[34] D.P. Roy, The Hadronic tau decay signature of a heavy charged Higgs boson at LHC, Phys. Lett. B 459 (1999) 607 [hep-ph/9905542] [SPIRES].

[35] T. Plehn, Charged Higgs boson production in bottom gluon fusion, Phys. Rev. D 67 (2003) 014018 [hep-ph/0206121] [SPIRES].

[36] S.-h. Zhu, Complete next-to-leading order QCD corrections to charged Higgs boson associated production with top quark at the CERN Large Hadron Collider, Phys. Rev. D 67 (2003) 075006 [hep-ph/0112109] [SPIRES].

[37] N. Kidonakis, Charged Higgs production via $b g \rightarrow t H^{-}$at the LHC, JHEP 05 (2005) 011 [hep-ph/0412422] [SPIRES].

[38] M. Beccaria, G. Macorini, L. Panizzi, F.M. Renard and C. Verzegnassi, Associated production of charged Higgs and top at LHC: the role of the complete electroweak supersymmetric contribution, Phys. Rev. D 80 (2009) 053011 [arXiv:0908.1332] [SPIRES]. 